\title{
A synergic framework for cyber-physical production systems in the context of Industry 4.0 and beyond
}

\author{
V.K. Chawla ${ }^{a^{*}}$, Surjit Angra ${ }^{b}$, Sandeep Suric and R.S. Kalra ${ }^{c}$
}

andira Gandhi Delhi Technical University for Women, Delhi, India

${ }^{b}$ National Institute of Technology, Kurukshetra, Haryana, India

${ }^{c}$ Delhi Institute of Tool Engineering (formerly TRTC-Indo Danish venture), Delhi, India

\section{H R O N I C L E}

Article history:

Received: September 11, 2018

Received in revised format: September 11, 2019

Accepted: December 12, 2019

Available online: December 12 2019

Keywords:

Big Data

Cloud Computing

Cyber-Physical Production Sys-

tems

Industry 4.0

Internet of Things

Synergic Framework

\section{A B S T R A C T}

With the inception of high-speed internet data services and ever-growing technical advancement in manufacturing technology, the integration of production systems and the internet of things to produce different types of jobs via cloud computing has become possible. The internet-enabled advanced automatic production systems can be referred to as the cyber-physical production systems (CPPS). The use of CPPS via cloud computing and the internet of things (IoT) can offer high productivity and high flexibility for the production of jobs in a dynamic production environment with varying specifications. The aim of this paper is to present a generalized synergic framework between different production facilities locating at different geographical locations to realize an energy-saving and efficient cyber-physical production system for the production of different types of jobs in the context of the industry 4.0 and beyond. In addition to the above, the study also identifies a need to address large scale multi-objective optimization issues to make the best decisions for different combinatorial production scenarios by using CPPS that are functioning in smart production facilities at different geographical locations.

C 2020 by the authors; licensee Growing Science, Canada.

\section{Introduction}

Industry 4.0 is known as the fourth industrial revolution globally (Gilchrist, 2016; Liao et al., 2017; Lu, 2017). Industry 4.0 consists of independent and autonomous machines that are highly automated and can be controlled by an automatic control via high-speed big data transmission through the internet. The machines used in the industry 4.0 can be referred to as cyber-physical production systems (CPPS), which are developed by integration of mechanical engineering, computer science, electronics engineering and information technology altogether (Verl et al., 2012; Vogel et al., 2014; Vogel et al., 2015). The CPPS, by using its artificial intelligence (AI) yields high throughput in a production facility (Kolberg \& Zühlke, 2015; Chryssolouris et al., 2016; Jiang et al., 2016; Roblek et al., 2017). The modern manufacturing 
system uses the internet of things (IOTs) feature, which is complemented with high-speed wireless networks. By using high-speed networking in the production systems, the production operations on different production systems can be conveniently synchronized to produce different types of jobs with high productivity (Wang et al., 2015; Wan et al., 2016; Jeschke et al., 2017). The operation and control of too many CPPS situated at different geographic locations call for an enormous amount of data transmission via cloud computing architecture. By using a cloud computing architecture and high-speed networking the CPPS can be operated and controlled without any human physical intervention from different geographical location (Monostori, 2014; Lee et al., 2014; Kühnle \& Bitsch, 2015; Schlechtendahl et al., 2015; Jiang et al. 2016; Karkalos et al., 2019; Sipsas et al., 2016). In order to yield high productivity from the cyber-physical production systems operating at different geographic locations, it is highly imperative to use an efficient communication network and cloud computing system architecture. An efficient synergic framework must be developed between different production facilities at different geographic locations to yield maximum productivity and best utilization of CPPS operational capabilities.

In industry 4.0, different production machines are inter-connected with each other and work in a collaborative manner (Lee et al., 2014; Xu et al., 2018). The production system uses an advanced data analysis and prediction tool to manage the big data associated with different machining operations and take a realtime efficient decision autonomously in a most timely manner to achieve the best production levels (Lee et al., 2014; Rojas et al., 2017; Wang and Wang, 2018). A multi-layer structural framework to make the manufacturing systems ready for industry 4.0 is proposed by Qin et al. (2016). The authors focused on different manufacturing scenarios and discussed the basic aspects of industry 4.0. Parallel development of information science, network science, computer science and production engineering and their impact on cyber-physical production systems (CPPS) is investigated by Monostori (2014). The authors investigated different challenges and issues to implement CPPS in industry 4.0. Chryssolouris et al. (2016) proposed a decision support system for line workers and maintenance workers in an emergency repair situation of CPPS for a production facility in the context of the industry 4.0. The aforesaid decision support system is observed to work on a multi-layer service-oriented architecture that integrates different sub-systems, sensors, information and data with each other. The authors used a case study to highlight the appropriateness of the proposed decision support system and new developments.

Wang et al. (2016) reported an integrated framework for the deployment of cloud communication for communication between work centers, material handling systems and other components in the industrial establishment. Authors focused on operational and control features of production systems in light of big data supported closed-loop systems. Various technical features and advantages of the proposed integrated framework are also highlighted in the study. Kolberg and Zühlke (2015) presented a combined approach of a lean production system and automation in the context of industry 4.0. The authors discussed all major aspects of industry 4.0 to link them and realize a lean production system. The authors also discussed a scheduling system for a KANBAN production system. Jiang et al. (2016) observed that the manufacturing sector is moving towards social factors namely mass-integration, socialization, servicing sector and in view of aforementioned social factors, authors proposed a social manufacturing approach for factory of future. The authors focused on the integration of CPPS and social media and proposed effective production strategies. In order to yield best productivity by evolving social innovation, the authors reported three main aspects namely operation, management and specifications. Hofmann and Rüsch (2017) observed industry 4.0 as an integrated industry of the future having the highest impact on production strategies for the production of different goods. The authors worked on the logistics management aspect associated with industry 4.0 and described different logistics issues and their impact on the functioning of industry 4.0. The authors considered decentralization, self-autonomy, effectiveness, and potential of just in time (JIT), just in sequence (JIS) and KANBAN for implementing them in the industry 4.0. Lee et al. (2015) mentioned that the cyber-physical systems (CPS) are closely connected and deployed in the industry 4.0. The performance of CPSs is closely monitored and integrated with other equipment to yield maximum productivity from them. The authors introduced a unified five-level system architecture for the CPS, to yield effective functioning of CPS in the industry 4.0. 
From the literature, it is evident that the CPPS can be efficiently used for the production of different types of jobs in the industry 4.0. Very limited research work is found in the development of an integrated framework for the production of different jobs at more than one production facility operating at different geographic locations. In order to full-fill the aforementioned research gap, a generalized cloud computing-based synergic framework between different smart production facilities situated at different geographical locations is introduced for the production of different types of jobs. The proposed framework is highly efficient in utilizing cyber-physical production systems for the production of different types of jobs.

\section{Problem descriptions}

The automatic production machines offer accuracy, precision, and fast production speed. The production machines can have variation in their features and production capabilities due to their varying specifications at operation level, integration level, intelligence level and production operation of a machine for a given operation command in a production cycle. The automation level and intelligence level of automatic production machines can be categorized on the basis of technology and hardware used in them. A high operation level in the production machines can be achieved by incorporating and using computer numeric control (CNC), program logic control (PLC) and other automatic features to deliver required machining operation efficiently with minimum human intervention. The machines with IoTs capabilities are referred to as cyber-physical production systems (CPPS). The CPPS works in a digital production environment and produces jobs in a highly efficient manner. The IOTs can be efficiently used for the integration of different production facilities operating from different geographic locations to boost up the production capabilities and optimize the overall production operations. A synergic framework for the efficient utilization of different CPPS operating with distributed control in the context of industry 4.0 is proposed in this study. The overall objective of the proposed synergic framework is to synchronize the production operations of different production facilities to yield a high level of flexibility and productivity in the production facilities which are operating at different geographic locations, for the production of different types of jobs in varying quantities. The pictorial representation is shown in figure 1 with following abbreviations.

\section{A synergic framework}

The cyber-physical production systems receive and transfer big data through machine sensors, machine control, and management systems via data transfer lines or wireless networks in the production facility. The data can be sent or received from outside of the production facility also. For example, transfer and receiving data from different vendors and customers. During the production operation, the real-time production data is transferred at high-speed every second by using a highly efficient data transfer network. The CPPS can send or receive the big data to an overall plant controller or to knowledge-based agents that initiate, integrate, synchronize and control the automatic production operations for all CPPS operating in a production facility. The efficiency of CPPS for different production operations significantly depends on the capability of high-speed data transfer and two-way communication channels between CPPS and the over-all plant controller.

In order to achieve an efficient production process, the CPPS must have the following features.

i) High-speed connectivity features to transfer and store real-time production data.

ii) Information feed-back function to analyze and take corrective actions.

iii) A high level of an intelligent management system.

iv) High computational speed and robust control.

v) High-reliability levels.

vi) Highly precise, accurate, repeatable and automatic for the performance of an assigned task. 


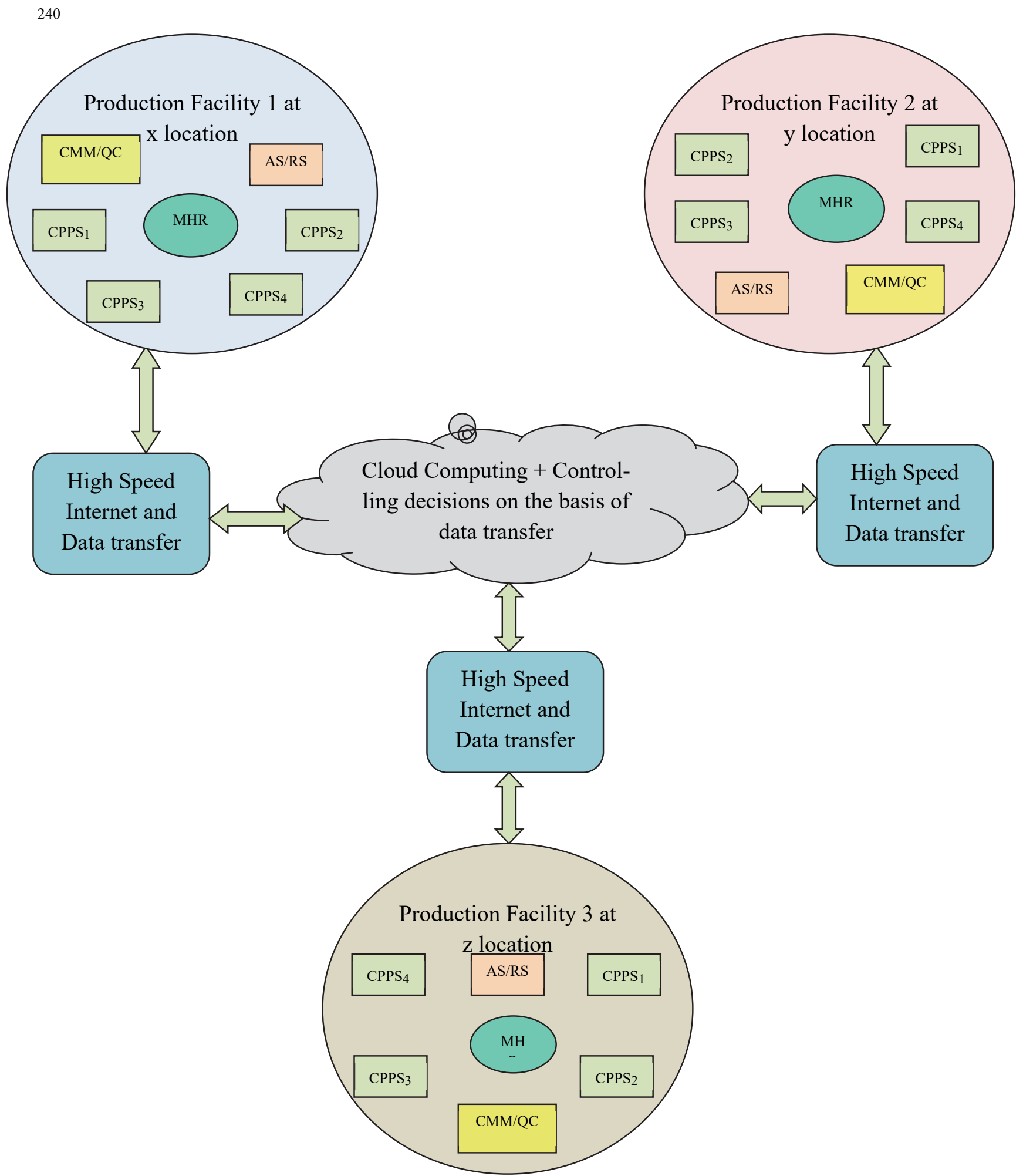

Fig. 1. Production of jobs at different locations

CMM: Coordinate measuring machine.

QC: Quality control.

CPPS: Cyber-physical production systems.
MHR: Material handling robot.

AS: Automatic storage.

RS: Retrieval system.

The CPPS with features like high connectivity, high computational power, and high intelligence feature offers best in class functional capabilities namely. The CPPS is highly flexible to adapt any kind of 
production schedule; the CPPS will become more adaptable to the integration with the production schedule of other machines or equipment functioning in the production facility. The functional capabilities, autonomy, availability, and reliability of CPPS increase significantly in vertical as well as in cross-functional domains. The high-speed internet facilitates the two-way communication capabilities between different components of CPPS at all levels, for example, sensor-sensor, actuator-sensor, actuator- object, object-mobile device, and mobile device-user, etc.

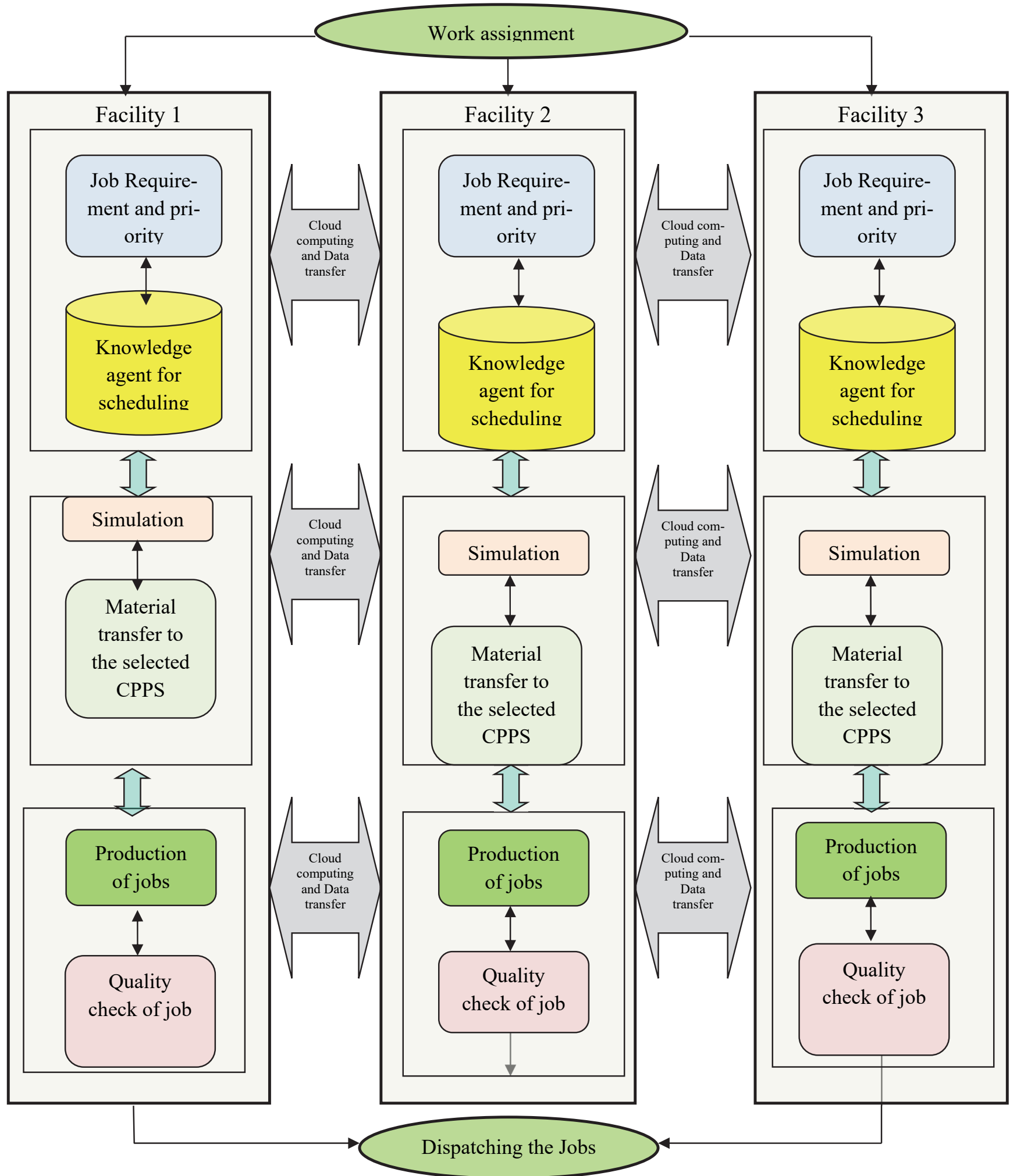

Fig. 2. A synergic framework for the production of jobs by the CPPS for the industry 4.0 
The machine learning and artificial intelligence can have a significant impact on the CPPS capabilities operating in the industry 4.0. A CPPS operating with intelligent production architecture can achieve maximum productivity and produces jobs at a very faster rate in comparison to any other advanced production system. Therefore, in order to have a fast and accurate decision by a CPPS, the knowledge-based agent is always desirable in software architecture. With the aforementioned capabilities, a CPPS makes intelligent decisions according to the production requirements. The cloud-based computing architecture along with intelligent decision-making capabilities improves the CPPS productivity and also improves the synchronizing capabilities of various CPPS during production operations in the production facilities operating at different geographical locations. A synergic framework for the production of jobs at three different production facilities in the context of industry 4.0 is delineated in figure 2 . In the proposed framework the production of the jobs is done on CPPS functioning at three different production facilities at different geographic locations. In order to control the production process, the production facilities are supported by cloud computing architecture and high-speed data transfer network. Initially, the work assignment is done the three production facilities, namely facility 1 , facility 2 and facility 3 . The work assignment is done on the basis of required production quantity and quality and the availability of idle resources in the aforementioned production facilities. Subsequently, the priorities and schedules of the production operations on CPPS are decided by using a knowledge-based agent for the production of jobs. Thereafter, the production process is simulated to search for the best possible combination of production sequences and available CPPS. On the basis of the output of the simulation, the raw material and semifinished jobs are transferred by using the material handling system at the different locations of the allocated CPPS. The production operations are carried out on the CPPS and the finished jobs are transferred to inspection centers. If the production quality of finished jobs is within the required dimensional tolerance limits, the jobs are stored and dispatched to the customer else segregation, rejection or rework on jobs is performed.

The cloud computing and high-speed data transfer feature benefits in fast two-way communications between the production facilities situated at different geographic locations. The production facilities are always updated about the real-time production conditions and utilization level of different machines and equipment that are operating in the production facilities. By using high-speed data transfer network the big data for production schedules and processes can be computed, analyzed and shared in a real-time manner. The information between different production facilities is shared regarding the job drawings, machining programs, reliability of machines, and utilization level of other production equipment, inventory levels, quality reports, and scrap details and updated in the cloud computing architecture via highspeed data transfer network. On the basis of accumulated information, the production operations of different production facilities for the production of different jobs can be synchronized and the uncertainty in production planning is minimized and a robust production schedule for all production facilities can be conveniently realized. A holistically developed production schedule can be implemented to minimize the makespan, maximize the throughput and achieve maximum utilization of all production facilities operating at different locations. The following main benefits can be achieved by adopting the proposed synergic framework.

i. The uncertainty in environmental factors, varying production loads and reliability issues are inevitable in any production process and make an adverse effect on the realization of production targets. In order to overcome the aforementioned issues, the use of CPPS is recommended which increases the adaptability, reliability and minimizes the idle cost in the production operations.

ii. The high level of automated production systems lowers down the human involvement in the production of the jobs. A high level of autonomy in the CPPS tenders the production of jobs in a clean environment and safe conditions. The use of CPPS reinforces sustainable development goals by optimum resource utilization, implementing global environmental policies, and developing a safe working environment. However, CPPS may also require some human input at some stage of the production process. Therefore, the level of automation infused in a CPPS is a deciding factor for human involvement in the functioning of any CPPS. 
iii. The CPPS can be controlled by a centralized controller or a CPPS can have a distributed control system. With high connectivity features and artificial intelligence, the self-decision making CPPS with the distributed control system is most preferred to use.

iv. The CPPS utilization can be maximized by their effective lateral, vertical and cross integration with other resources, services, features, and production facility system architecture. The CPPS offers the best of their services in their domain with their inter-production facility and intra-production facility integration features. By integration of CPPS with other production utilities the intelligence, knowledge, information sharing, and adaptability of CPPS significantly increases.

v. The IoTs feature in the CPPS enables user-friendly and energy-efficient smart manufacturing. The IoTs based smartly designed and intelligence equipped production centers, autonomous vehicles, robots, and coordinate measuring machines (CMMs) improve and optimize the overall production process. The smart CPPS can develop its production schedule autonomously and adopt it in a real-time manner.

A synergic framework for IoTs based smart production facilities and its implementation in the context of industry 4.0 is proposed. The high level of industry 4.0 represents the utilization of a high level of computational power for the effective integration of production facilities operating at different geographic locations. The proposed framework can yield the energy-efficient production of jobs. Industry 4.0 uses efficient intelligent algorithms to produce a variety of jobs with high quality. The aforementioned framework enlightens a roadmap for the development of future production facilities in view of industry 4.0 and beyond.

\section{Conclusions}

In this study, a cloud computing-based energy-saving and efficient synergic framework between different CPPS is presented. In the proposed synergic framework, the CPPS operates at different production facilities which are located at different geographic locations. Numerous benefits associated with the proposed synergic framework in the production of different types of jobs are also discussed. It is observed that the IoT based production facilities harness the best utilization from production resources in a dynamic and uncertain production environment. Additionally, the proposed synergic framework advocates extensive use of artificial intelligence-based computation and distributed control architecture to solve the large scale multi-objective production scheduling issues in the smart production facilities. The research can be extended to solve large scale multi-objective optimization problems for parallel computation of all CPPS and to search the best solutions for their efficient scheduling while operating at different geographical locations. In order to maximize the throughput of different production facilities operating at different locations, the production factors namely job mix, production priorities, material transfer operations, automatic storage and retrieval of jobs, logistics and reliability of CPPS, etc. can also be considered.

\section{References}

Gilchrist, A. (2016). Industry 4.0: the industrial internet of things. Apress.

Hofmann, E., \& Rüsch, M. (2017). Industry 4.0 and the current status as well as future prospects on logistics. Computers in Industry, 89, 23-34.

Jeschke, S., Brecher, C., Meisen, T., Özdemir, D., \& Eschert, T. (2017). Industrial internet of things and cyber manufacturing systems. In Industrial Internet of Things (pp. 3-19). Springer, Cham.

Jiang, P., Ding, K., \& Leng, J. (2016). Towards a cyber-physical-social-connected and service-oriented manufacturing paradigm: Social Manufacturing. Manufacturing Letters, 7, 15-21.

Karkalos, N. E., Markopoulos, A. P., \& Davim, J. P. (2019). Computational Methods for Application in Industry 4.0. Springer International Publishing.

Kolberg, D., \& Zühlke, D. (2015). Lean automation enabled by industry 4.0 technologies. IFAC-Papers On Line, 48(3), 1870-1875. 
Kühnle, H., \& Bitsch, G. (2015). Foundations \& principles of distributed manufacturing (Vol. 422). Berlin: Springer.

Lee, J., Kao, H. A., \& Yang, S. (2014). Service innovation and smart analytics for industry 4.0 and big data environment. Procedia Cirp, 16, 3-8.

Lee, J., Bagheri, B., \& Kao, H. A. (2015). A cyber-physical systems architecture for industry 4.0-based manufacturing systems. Manufacturing letters, 3, 18-23.

Liao, Y., Deschamps, F., Loures, E. D. F. R., \& Ramos, L. F. P. (2017). Past, present and future of Industry 4.0-a systematic literature review and research agenda proposal. International journal of production research, 55(12), 3609-3629.

Lu, Y. (2017). Industry 4.0: A survey on technologies, applications and open research issues. Journal of Industrial Information Integration, 6, 1-10.

Monostori, L. (2014). Cyber-physical production systems: Roots, expectations and R\&D challenges. Procedia Cirp, 17, 9-13.

Qin, J., Liu, Y., \& Grosvenor, R. (2016). A categorical framework of manufacturing for industry 4.0 and beyond. Procedia Cirp, 52, 173-178.

Roblek, V., Meško, M., \& Krapež, A. (2016). A complex view of industry 4.0. Sage Open, 6(2).

Rojas, R. A., Rauch, E., Vidoni, R., \& Matt, D. T. (2017). Enabling connectivity of cyber-physical production systems: a conceptual framework. Procedia Manufacturing, 11, 822-829.

Schlechtendahl, J., Keinert, M., Kretschmer, F., Lechler, A., \& Verl, A. (2015). Making existing production systems Industry 4.0-ready. Production Engineering, 9(1), 143-148.

Sipsas, K., Alexopoulos, K., Xanthakis, V., \& Chryssolouris, G. (2016). Collaborative maintenance in flow-line manufacturing environments: An Industry 4.0 approach. Procedia Cirp, 55, 236-241.

Verl, A., Lechler, A., \& Schlechtendahl, J. (2012). Glocalized cyber physical production systems. Production Engineering, 6(6), 643-649.

Vogel-Heuser, B., Diedrich, C., Pantförder, D., \& Göhner, P. (2014, July). Coupling heterogeneous production systems by a multi-agent based cyber-physical production system. In 2014 12th IEEE International Conference on Industrial Informatics (INDIN) (pp. 713-719). IEEE.

Vogel-Heuser, B., Lee, J., \& Leitão, P. (2015). Agents enabling cyber-physical production systems. atAutomatisierungstechnik, 63(10), 777-789.

Wan, J., Tang, S., Shu, Z., Li, D., Wang, S., Imran, M., \& Vasilakos, A. V. (2016). Software-defined industrial internet of things in the context of industry 4.0. IEEE Sensors Journal, 16(20), 7373-7380.

Wang, L., Törngren, M., \& Onori, M. (2015). Current status and advancement of cyber-physical systems in manufacturing. Journal of Manufacturing Systems, 37, 517-527.

Wang, S., Wan, J., Li, D., \& Zhang, C. (2016). Implementing smart factory of industry 4.0: an outlook. International Journal of Distributed Sensor Networks, 12(1), 3159805.

Wang, L., \& Wang, X. V. (2018). Cloud-Based Cyber-Physical Systems in Manufacturing. Springer International Publishing.

Xu, L. D., Xu, E. L., \& Li, L. (2018). Industry 4.0: state of the art and future trends. International Journal of Production Research, 56(8), 2941-2962.

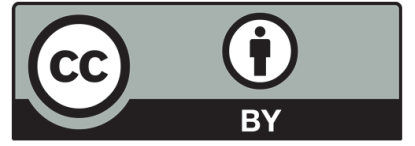

(C) 2020 by the authors; licensee Growing Science, Canada. This is an open access article distributed under the terms and conditions of the Creative Commons Attribution (CC-BY) license (http://creativecommons.org/licenses/by/4.0/). 